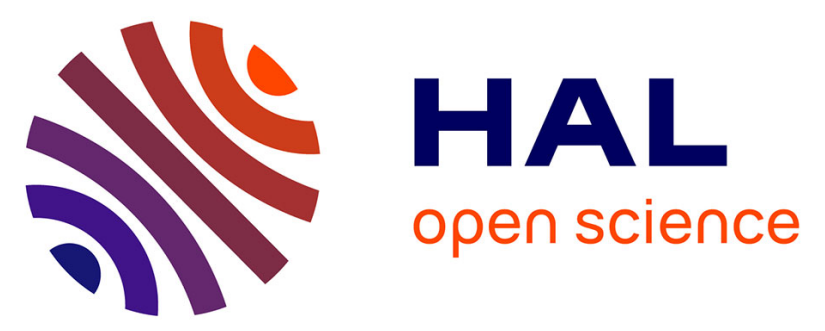

\title{
A multi-level approach to investigate the control of an input device: application to a realistic pointing task
}

Hugo Loeches de La Fuente, Guillaume Rao, Jean-Christophe Sarrazin, Eric

Berton, Laure Fernandez

\section{- To cite this version:}

Hugo Loeches de La Fuente, Guillaume Rao, Jean-Christophe Sarrazin, Eric Berton, Laure Fernandez. A multi-level approach to investigate the control of an input device: application to a realistic pointing task. Ergonomics, 2014, 57 (9), pp.1380-1396. 10.1080/00140139.2014.921330 . hal-01442057

\section{HAL Id: hal-01442057 \\ https://hal.science/hal-01442057}

Submitted on 15 Nov 2017

HAL is a multi-disciplinary open access archive for the deposit and dissemination of scientific research documents, whether they are published or not. The documents may come from teaching and research institutions in France or abroad, or from public or private research centers.
L'archive ouverte pluridisciplinaire HAL, est destinée au dépôt et à la diffusion de documents scientifiques de niveau recherche, publiés ou non, émanant des établissements d'enseignement et de recherche français ou étrangers, des laboratoires publics ou privés. 
This article was downloaded by: [Aix-Marseille Université], [Guillaume RAO]

On: 03 J une 2014, At: 06:43

Publisher: Taylor \& Francis

Informa Ltd Registered in England and Wales Registered Number: 1072954 Registered office: Mortimer House, 37-41 Mortimer Street, London W1T 3J H, UK

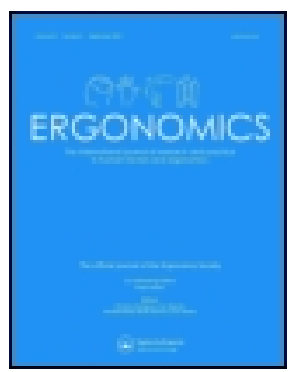

\section{Ergonomics}

Publication details, including instructions for authors and subscription information:

http:// www. tandfonline.com/loi/terg20

\section{A multi-level approach to investigate the control of an input device: application to a realistic pointing task}

Hugo Loeches De La Fuente ${ }^{a}$, Guillaume Rao ${ }^{a}$, J ean-Christophe Sarrazin ${ }^{b}$, Eric Berton ${ }^{a} \&$ Laure Fernandez ${ }^{a}$

${ }^{a}$ Aix-Marseille Université, CNRS, ISM UMR 7287, 13288, Marseille, France

${ }^{\mathrm{b}}$ Office National d'Etudes et de Recherches Aérospatiales, département Commande des systèmes et dynamique du vol (DCSD), Salon de Provence, France

Published online: 30 May 2014.

To cite this article: Hugo Loeches De La Fuente, Guillaume Rao, J ean-Christophe Sarrazin, Eric Berton \& Laure Fernandez (2014): A multi-level approach to investigate the control of an input device: application to a realistic pointing task, Ergonomics, DOI: 10.1080/00140139.2014.921330

To link to this article: http:// dx. doi.org/ 10.1080/00140139.2014.921330

\section{PLEASE SCROLL DOWN FOR ARTICLE}

Taylor \& Francis makes every effort to ensure the accuracy of all the information (the "Content") contained in the publications on our platform. However, Taylor \& Francis, our agents, and our licensors make no representations or warranties whatsoever as to the accuracy, completeness, or suitability for any purpose of the Content. Any opinions and views expressed in this publication are the opinions and views of the authors, and are not the views of or endorsed by Taylor \& Francis. The accuracy of the Content should not be relied upon and should be independently verified with primary sources of information. Taylor and Francis shall not be liable for any losses, actions, claims, proceedings, demands, costs, expenses, damages, and other liabilities whatsoever or howsoever caused arising directly or indirectly in connection with, in relation to or arising out of the use of the Content.

This article may be used for research, teaching, and private study purposes. Any substantial or systematic reproduction, redistribution, reselling, loan, sub-licensing, systematic supply, or distribution in any form to anyone is expressly forbidden. Terms $\&$ Conditions of access and use can be found at http:// www.tandfonline.com/page/terms-and-conditions 


\title{
A multi-level approach to investigate the control of an input device: application to a realistic pointing task
}

\author{
Hugo Loeches De La Fuente ${ }^{\mathrm{a} *}$, Guillaume Rao ${ }^{\mathrm{a}}$, Jean-Christophe Sarrazin ${ }^{\mathrm{b}}$, Eric Berton ${ }^{\mathrm{a}}$ and Laure Fernandez ${ }^{\mathrm{a}}$ \\ ${ }^{a}$ Aix-Marseille Université, CNRS, ISM UMR 7287, 13288, Marseille, France ${ }^{b}$ Office National d'Etudes et de Recherches Aérospatiales, \\ département Commande des systèmes et dynamique du vol (DCSD), Salon de Provence, France
}

(Received 15 October 2013; accepted 22 April 2014)

\begin{abstract}
This study investigates the subjects' performance during realistic conditions of control of a joystick. An adapted reciprocal aiming task consisting in driving a virtual vehicle along a slalom course as fast as possible was performed while accuracy constraints were manipulated. Realistic dynamical Interface Screen Relationship between the joystick displacements and the displacements of the vehicle was simulated. Vehicle displacements and motor activity (muscle activity and joint kinematics) were recorded. The results highlighted the applicability of the Fitts' law to more realistic conditions where the use of an input device is performed in an intensive control situation. Besides, biomechanical results suggested that neuromuscular responses were different regarding the direction of movement, whereas the performance at a behavioural level were not affected. Thus, this study demonstrates the interest in considering two different aspects of the user's performance (behavioural and biomechanical ones) to make a better agreement between the device design and users' needs.
\end{abstract}

Practitioner Summary: This study considered two different aspects of the subject's performance in a realistic situation of speed-accuracy trade-off: the behavioural and motor activity. The necessity for the design of the future ergonomics pointing devices to meet the expectations of the neuromuscular system in order to facilitate their uses is highlighted.

Keywords: input device; user performance; motor control; biomechanics; multi-level analysis

\section{Introduction}

Input devices in human-computer interaction (HCI) are constantly renewed to provide the best possible agreement between the user's capabilities and their resulting 'virtual' activity on the screen. Choosing input devices for interactive systems that best suit user's needs remains a challenge, especially with the increasing number of devices available. Zhai (1995) classified the input devices as isotonic, isometric or elastic. Isotonic devices are free-moving and use position for input such as a computer mouse. Isometric devices do not move and use force for input such as the TrackPoint. Between isotonic and isometric devices are elastic devices, where resistance increases with displacement. Elastic devices can use either position or force for input. One example of elastic device is the two degrees of freedom (DOF) joystick that is widely used for video games or working situations such as industrial control, aviation or surgical robotics. The relationship between the inclination of the joystick in degrees (effector space) and the displacement of the pointer in pixel (task space) can be defined as an Interface Screen Relationship (ISR).

Contemporary computer-based user interfaces are designed around direct manipulation of objects on screen, with an input device being used to move the cursor to items, and to click, drag or manipulate them. The speed, accuracy and efficiency of pointing are therefore extremely important to the overall effectiveness of the computer use. However, for pointing tasks, precision requirements affect completion time. Pioneering studies on fast and accurate pointing movements have shown that movement speed decreases when accuracy constraints increase (Woodworth 1899). Consequently, input devices users face a speed/accuracy trade-off: the faster the reaching movement, the more likely that error occurs. In order to evaluate users' performance when engaged in such task, quantitative variables, chosen by the designers of the computer input devices, are based on the Fitts' performance model (Fitts 1954). According to Fitts' law, the time required to perform an accurate pointing movement depends on the index of difficulty (ID) defined as the logarithm of the ratio between target distance and tolerance (i.e. target size). This law predicts the movement time (MT) required to point a target of size $W$ at a distance $D$ :

$$
\mathrm{MT}=a+b \times \log _{2}(2 D / W)
$$

\footnotetext{
*Corresponding author. Email: hugo.loeches-de-la-fuente@univ-amu.fr
} 
In Equation (1), the term $\log _{2}(2 D / W)$ represents the ID of the task and is expressed in bits. The higher the value of ID, the more difficult the task is.

Fitts' law is known as one of the most robust laws governing motor control. Recently, this law has been declined for several specific situations such as underwater movements (Hoffmann and Chan 2012), moving about obstructions (Hoffmann and Sheikh 2012) and 2D or 3D target aiming (Hoffmann, Drury, and Romanowski 2011). In HCI literature, authors have found that the MT of a cursor controlled with different input devices (a mouse, step keys, text keys or a joystick) on a computer screen also followed Fitts' law (Card, English, and Burr 1978). Indeed, Card, English, and Burr (1978) first realised that Fitts' paradigm, which consists in pointing targets as fast and as accurately as possible, could be used as a methodological tool for HCI researchers to evaluate the use of most input devices performance (joystick, mouse, trackball, etc.). In this literature, the index of performance (IP) combining both speed and accuracy (Fitts and Peterson 1964) is regularly analysed (Soukoreff and MacKenzie 2004; Zhai 2004) and used to quantify pointing performance (Hoffmann 2012; Isokoski and Raisamo 2002; MacKenzie 1992a; MacKenzie and Jusoh 2001; Wright and Lee 2013). IP, considered as a fundamental metric, is computed as:

$$
\mathrm{IP}=\frac{\mathrm{ID}_{e}}{\mathrm{MT}}
$$

where $\mathrm{ID}_{\mathrm{e}}$ is the effective index of difficulty adjusted by using effective width of the target $\left(W_{\mathrm{e}}\right)$ based on the actual endpoint distribution:

$$
\mathrm{ID}_{e}=\log _{2}\left(\frac{2 D}{W_{e}}\right)
$$

Using effective width of the target incorporates the variability observed in human performance (MacKenzie 1992b). The value of IP has been recognised by both academic and industry researchers, as it has been codified in the ISO standard describing the evaluation of pointing devices (ISO 2002). As a consequence, Fitts' paradigm is used to quantify performance in order to compare and to evaluate new input devices.

While pointing tasks obey speed-accuracy trade-off rules, the ISR influences the user's aiming performance. If the gain induced by the ISR is too weak, the velocity of the cursor, controlled by the user through the joystick, is going to decrease. If the gain is too high, the accuracy of the cursor's position is going to be lower (Casiez et al. 2008). A criticism that one may formulate from the previous cited studies is that the performance was only analysed through the IP without taking into account the kinematic organisation of end-point movement. In this perspective, Mottet and Bootsma (1999) investigated the reciprocal form of the Fitts paradigm in order to understand the control mechanisms that govern goal-directed movements. They demonstrated that kinematic organisation differs depending on the level of difficulty. Classically, easy reciprocal aimings (low IDs) present a harmonic or sinusoidal feature, with a symmetrical velocity profile. However, as the difficulty of the task increases, the kinematic pattern loses its sinusoidal characteristics with the peak of velocity occurring earlier, resulting in a longer deceleration phase (Boyle et al. 2012; Elliott et al. 2010; Fernandez and Bootsma 2004; Guiard 1993; Mottet and Bootsma 1999). In line with these problems, Fernandez and Bootsma (2008) investigated Fitts' experimental paradigm in the reciprocal version, to test the consequences of a non-linear ISR on the kinematic organisation of movement. This non-linear ISR corresponded to a logistic relationship between the position of the stylus on a graphic tablet (effector space) and the position of the cursor on the screen (task space). Results showed that the logistic ISR allowed for more rapid and more harmonic movements for all levels of task difficulty, without loss of end-point precision. Thus, the non-linear ISR between effector space (i.e. joystick) and task space (i.e. screen) helped the subjects in mastering the task. Similarly, Casiez et al. (2008) investigated the users' performance in a reciprocal Fitts' task by varying the ISR based on modifications of pointer accelerations (PA). Results showed that MT with modifications on PA was 3.3\% lower than a constant ISR, inducing a higher pointing performance. Both studies provided evidence of the influence of non-linear or non-uniform ISR on user's performance.

However, most of the cited investigations are laboratory studies, whereas nowadays uses of input devices are increasingly dedicated to intensive control situations where realistic conditions of use are at work (aviation, industrial control or video games). Such situations require controlling the movements of real or virtual objects characterised by inertial characteristics. Thereby, users control the forces applied to the object by displacing the input device in the effector space. The current ISRs are mostly based on massless objects that are moved on the screen, whereas everyday object manipulation is subject to gravity and inertial constraints. Nevertheless, as far as we know, the current literature in the ergonomics research field has not focused on realistic inertial relationships between the task and the effector spaces.

As the performance depends on an appropriate use of our motor skills when controlling an input device, it is also important to consider motor activity in order to investigate more precisely the users needs. Indeed, muscle activity has been 
reported to play an important role in the control of precise movements. It is well known that during goal-directed movements the simultaneous activation of muscle groups having opposite functions plays a stabilisation role: for high accuracy requirement, kinematic variability can be controlled by increasing co-activation of muscles (Gribble et al. 2003; Huysmans et al. 2012; Osu et al. 2004; van Galen and de Jong 1995). Huysmans et al. (2012) showed that the participants performing a tracking task with a pen on a graphic tablet increased the simultaneous activation of wrist extensor and flexor muscle groups when the size of the target was reduced. If we wish to investigate users' needs as a whole, we should focus on the relationship between motor activity during the use of an input device in the effector space and the resulting behaviour in the task space. As of now, the HCI literature is scarce in studies that consider this relationship. This lack is especially observed for intensive control situations that are highly demanding for the users (fatigue induced by the motor action, chronic musculoskeletal pain relative to a non-ergonomics use, etc.).

In the present study, we propose to analyse the user's performance while manipulating an elastic device under realistic inertial conditions using a dynamical model characterising the relationship between the positions in the effector space and the positions in the task space. Our first hypothesis states that Fitts' law will be preserved in our task with an increase of ID affecting the user's control in both the task and the effector spaces. Classically, we expect an increase of MT and a loss of the sinusoidal characteristics of the kinematic patterns with increasing task difficulty. We also hypothesise that an increase of ID will induce a decrease of end-point variability in task space $\left(W_{\mathrm{e}}\right)$ resulting in an increase of the effective index of difficulty $\left(\mathrm{ID}_{\mathrm{e}}\right)$. Finally, we hypothesise that the muscular parameters will be affected by the accuracy constraints with an increase in co-activation as the ID increased. Taken together, these results would lead to a better understanding of the users' needs during the manipulation of an elastic pointing device subjected to a realistic ISR.

\section{Method}

\subsection{Participants}

Participants were 10 right-handed volunteers ( 2 women and 8 men, mean age 27). All participants had no previous history of upper extremity musculoskeletal disorders and all reported normal or corrected to normal vision. All participants were experienced computer users but none of the subjects had prior experience with the interface used in this study. All participants signed an informed consent according to university guideline that was approved by the Aix-Marseille University ethics committee.

\subsection{Tasks}

The participants had to drive a vehicle in a virtual environment using a two DOF elastic joystick (Logitech Extreme 3D Pro), as in a video game. The vehicle had neither length nor width and the vehicle position corresponded to the participants' point of view. Participants used the joystick to control the displacements of their own point of view. The displacements of the vehicle (i.e. the participants' point of view) in the horizontal plane along medio-lateral and longitudinal axes were controlled. Along each axis, identical dynamical relationships between the displacement of the joystick and the displacements of the vehicle were introduced (see the experimental set-up section and Loeches De La Fuente et al. [2014] for detail).

The participants were required to cross successively and as quickly as possible 22 doors presented in the virtual environment. We left out four doors at the beginning and six doors at the end in order to analyse 10 cycles with stable performance for each trial. A visual feedback was displayed on the screen at the end of each trial, presenting the percentage of successfully crossed doors. Whatever the experimental condition, the trial was considered successful if the rate of missed doors (i.e. error rate) was strictly less than $25 \% .^{1}$

During the familiarisation phase, the participants were informed that a minimum of $75 \%$ of doors had to be crossed for each trial to be successful. During the experimental phase, the trial was immediately redone if the minimum performance level required was not attained until the error criterion was met. A 1-minute rest between each trial (succesfull or not) was allowed.

The virtual environment where the task is defined and in which the vehicle moves will further be referred to as the task space, while the space in which the participant's upper limb moves will be referred to as the effector space.

\subsection{Experimental design}

Each condition was characterised by a quantified ID. ID $=\log _{2}(2 D / W)$, where $D$ is the distance between the centre of two successive doors (Figure 1) along the medio-lateral axis and $W$ is the width of the doors (i.e. the tolerance of the spatial precision). Because the accuracy constraints (i.e. $W$ ) are known to influence both the kinematic organisation of movement (Fernandez and Bootsma 2008; Guiard 1993; Mottet and Bootsma 1999) and muscle co-activation (Gribble et al. 2003; Laursen, Jensen, and Sjogaard 1998; Osu et al. 2004; Visser et al. 2004), task difficulty was manipulated through the width 

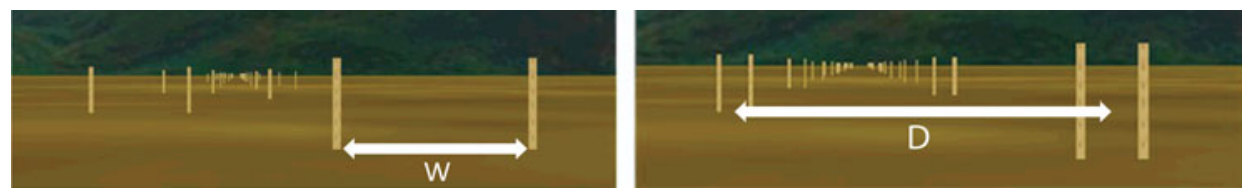

Figure 1. Task space visualisation representing the successive doors in the virtual environment.

Note: This visualisation corresponds to the subjects' point of view during the experimentation. $\mathrm{D}$ is the distance between the centre of two successive doors and $W$ is the inter-door distance. The parameter $W$ was tuned to obtain different index of task difficulty.

of the doors in the current study, while distance $D$ remained unchanged across conditions. Five widths $W(5.00,3.20,2.22$, 1.63 and $1.25 \mathrm{~m})$ and one distance $D(10.00 \mathrm{~m})$ were used.

The different values of the $W$ parameter rendered a total of five experimental conditions with ID ranging from 2 to 4 bits with a 0.5 bit increment. Such ID values, considered as low in classical Fitts' law studies, were determined regarding the features of the proposed task (2D displacements of the vehicle and non-linear ISR).

The experimental phase was constituted by eight trials of each of the different ID conditions. The order of the conditions was randomised to avoid any learning effect.

\subsection{Experimental set-up}

The following experimental set-up was used to record the vehicle position in the task space and the participant's motor activity from the 3D displacements of the upper limb segments and the electromyographic (EMG) activities in the effector space.

\subsubsection{Task space}

The virtual environment in which the vehicle was driven was simulated in real time with a dedicated software programme (ICE) developed in the laboratory and running on a laptop. The virtual environment (task space) presented the vehicle position and successive doors (Figure 1).

The displacements of the vehicle were characterised by a dynamical ISR simulating both inertia and air resistance (drag). This dynamical model is described by:

$$
\sum_{i} \vec{F}_{i}=m \vec{a}
$$

where $\vec{F}$ is the external force exerted on the vehicle. $m$ is the mass of the vehicle ( $\mathrm{m}=94 \mathrm{Kg}$ ).

In this study, the reference vehicle was an unmanned aerial vehicle (UAV).

$\vec{a}$ corresponds to the acceleration of the centre of mass of the vehicle, with

$$
\sum_{i} \vec{F}_{i}=\vec{F}_{A}-\vec{F}_{D}
$$

where $\vec{F}_{A}$ is the force of advancement given to the vehicle depending on the angular position $(\theta)$ of the joystick along each axis (medio-lateral and longitudinal axes). The relationship between the angular position of the joystick and $F_{A}$ along each axis was linear with $\vec{F}_{A}$ being proportional to angular position in the effector space. This relationship was formalised by:

$$
\overrightarrow{F_{A}}=\left(\begin{array}{c}
F_{a x} \\
F_{a y}
\end{array}\right)=k\left(\begin{array}{c}
\theta_{x} \\
\theta_{y}
\end{array}\right),
$$

where $F_{a x}$ is the force of advancement on the longitudinal axis, $F_{a y}$ is the force of advancement on the medio-lateral axis, $\theta_{x}$ is the angular position of the joystick around the medio-lateral axis, $\theta_{y}$ is the angular position of the joystick around the longitudinal axis, $k$ represents a linear gain. In the present experiment, $k$ was set to $1 . \theta_{x}$ and $\theta_{y}$ were both normalised and bounded within the interval $[-1,+1]$. Consequently, $F_{a x}$ and $F_{a y}$ were also included within the interval $[-1,+1]$. 
$\overrightarrow{F_{D}}$ is the drag force that appears along a direction opposite to the vehicle velocity. The drag force was formalised by:

$$
\overrightarrow{F_{D}}=\left(\begin{array}{l}
F_{d x} \\
F_{d y}
\end{array}\right)=\frac{1}{2} \rho A C_{D}\left(\begin{array}{c}
v_{x}^{2} \\
v_{y}^{2}
\end{array}\right),
$$

where $F_{d x}$ is the drag force on the longitudinal axis and $F_{d y}$ is the drag force on the medio-lateral axis. $v_{x}$ is the vehicle velocity on the longitudinal axis and $v_{y}$ is the vehicle velocity on the medio-lateral axis.

Velocities along each axis were scalar quantities in metres per second, $\rho$ is the air density in kilogram per cubic metre, $A$ is the reference area in square metre (area of the orthogonal projection of the vehicle on a plane perpendicular to the direction of motion) and $C_{D}$ is the dimensionless drag coefficient of the moving vehicle. In the present experiment, the product $\mathrm{AC}_{D}$ was equal to 0.254 representing the air penetration coefficient of the vehicle (UAV).

Along each axis of displacement in the task space and for each time step, a double time integration was applied to the vehicle acceleration data to obtain vehicle position (Equation (8) and Equation (9)).

$$
\begin{aligned}
& x(t)=\iint a_{x}(t) d t^{2}, \\
& y(t)=\iint a_{y}(t) d t^{2},
\end{aligned}
$$

where $x(t)$ and $a_{x}(t)$ are respectively the vehicle position and acceleration on the longitudinal axis at the time $t$ and $y(t)$ and $a_{y}(t)$ are respectively the vehicle position and acceleration on the medio-lateral axis at the time $t$.

The simulated environment was displayed on a LCD monitor $(20.5 \mathrm{~cm} \times 33 \mathrm{~cm}, 1440 \times 900$ pixels resolution). The monitor was positioned on a table in front of the participants at $50 \mathrm{~cm}$ from their trunk and the scene was displayed in an egocentric frame of reference with a horizontal field of view of $66^{\circ}$.

The position of the vehicle along the medio-lateral axis was recorded at $75 \mathrm{~Hz}$.

\subsubsection{Effector space}

The participants were seated with their knees at $90^{\circ}$ flexion. The location of the joystick on the table was adjustable to take into account the anthropometric characteristics of the participants. Thus, for each participant holding the joystick in its central position, the forearm was parallel to the table plane, the elbow was flexed at $90^{\circ}$ and the wrist was in neutral position (Figure 2).

The 3D postures of the upper limb segments were recorded by six cameras (Vicon Motion System, Lake Forest, CA) at a $60 \mathrm{~Hz}$ frequency. Five reflective markers were attached to the acromion (AC), the lateral epicondyle (LE), the centre of third metacarpal bone (C3), the distal head of third metacarpal bone (T3) and the distal head of second metacarpal bone (T2) (Wu et al. 2005). Surface EMG of pronator teres (PT) and brachioradialis (BR) muscles was recorded at $1980 \mathrm{~Hz}$ using a BIOPAC MP150 system with Ag/Ag-Cl bipolar surface electrodes (Skintact model FS 501, Innsbruck, Austria). Electrodes placement and locations were suggested by the SENIAM recommendations (Hermens et al. 2000).

Maximum voluntary contractions (MVC) of PT and BR muscles were recorded before the experimental phase using a fixed handle. Participants were instructed to apply as much force as possible on the handle in the two directions corresponding to the muscular actions of the two muscles (to the left and to the right). For each direction, three measurements of 3 seconds were achieved. Verbal encouragements were given to ensure maximum effort. A 1-minute rest was given between recordings.

All data (position of the vehicle, the 3D kinematics of the upper limb segments and the EMG activity) were recorded by synchronising the ICE software programme, the Vicon motion capture system and the BIOPAC MP150 system.

\subsection{Data processing}

In the present study, the virtual scene was composed of 30 doors to cross. A cycle corresponded to the data recorded between three successive doors and the participants performed 15 cycles per trial. We left out four doors at the beginning and six doors at the end in order to analyse 10 cycles with stable performance for each trial, thus resulting in 80 cycles per ID condition.

Each cycle was separated in two Directions of Movement (right- and left-directed), and for each direction of movement, all the recorded data were processed in order to obtain an average half cycle for each participant and each condition (ID). 


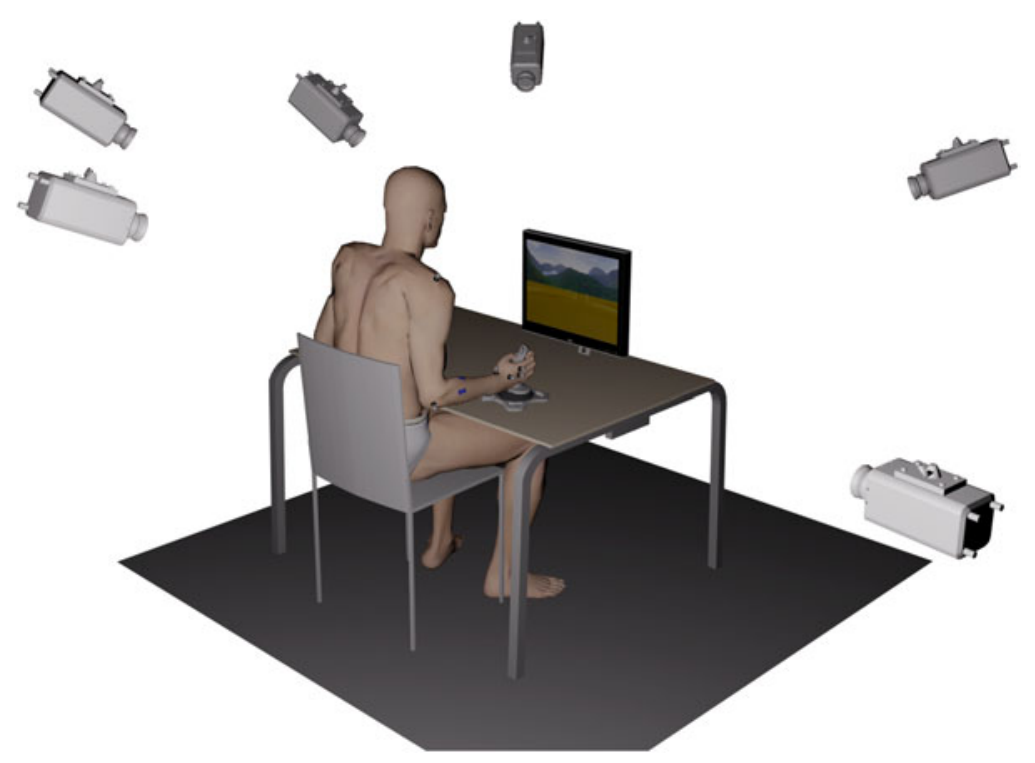

Figure 2. Experimental set-up visualisation.

Note: Five reflective markers and five surface electrodes (two for each analysed muscle and one for reference) were positioned on the right upper limb of the subject.

We used Eulerian angles method (Wu et al. 2005) to compute joint kinematics (forearm pronation/supination angle) from the reflective markers 3D positions. Velocity data were computed by deriving position against time in both the task and the effector spaces. In the task space, the medio-lateral vehicle position was derived against time. In the effector space, the forearm pronation/supination angle was derived against time. Position and velocity half cycles were rescaled within the interval $[-1,+1]$ and normalised in time (see Mottet and Bootsma [1999] for details on method). From position signal in task space and effector space, reversal points were then identified in order to distinguish right and left movements independently. Finally, the average position and velocity half cycles were obtained in task and effector spaces by averaging the corresponding half-cycle profiles.

For each data set (MVC and trials), EMG signals were band pass filtered from 20 to $400 \mathrm{~Hz}$, full-wave rectified and low pass filtered (Butterworth 2nd order, $6 \mathrm{~Hz}$ cut-off frequency). The maximum EMG value from the three MVCs was determined for each subject in each muscle and used to normalise the remaining EMG signals recorded during the experiment by expressing the EMG signal in percentage of the maximum EMG value. For each normalised muscle activity, right and left movements were distinguished independently using the reversal points of the position signal detected in effector space. We obtained average halfcycle profiles of each muscle activity by averaging the resulting half-cycle profiles.

For each trial, an average half cycle was obtained for all the data. Thus, we obtained a single average half cycle by averaging the eight half cycles. The dependent variables were computed from the average half cycles for each condition (ID) and each direction of movement.

\subsubsection{Dependent variables}

For the dependent variables used, the acronyms are respectively related to task or effector space by the last letter $T$ or $E$.

The aim of our analysis was to focus on a speed-accuracy trade-off by using an adapted reciprocal Fitts' task. In the task space, the different dependent variables were consequently computed from the medio-lateral vehicle position and velocity in the same way that reciprocal 1D displacements of the cursor on the screen are analysed in classical Fitts' law studies. Movement time (MTT) was defined as the average half-cycle time of medio-lateral vehicle position. Following segmentation of the movement based on movement reversals, MTT was calculated as the average time between two movement reversals. Time to peak velocity (TPVT) corresponds to the time (in percentage of the half-cycle time for both left and right movements) when the maximum velocity was achieved. We also computed the effective width of the target $\left(W_{\mathrm{e}}\right)$ that was defined as 1.96 times the standard deviation of the actual end-point distribution in task space (Huys et al. 2010; Welford 1968) with the end-point distribution corresponding to the medio-lateral vehicle position when crossing the door. $W_{\mathrm{e}}$ was used to compute $\mathrm{ID}_{\mathrm{e}}$ (see Equation (3)). Finally, the IP was computed from $\mathrm{MT}$ and $\mathrm{ID}_{\mathrm{e}}$ using Equation (2). Since the vehicle position was only considered as a single point (no width nor length for the displayed vehicle) along the lateral and the longitudinal axes, we chose not to investigate collision with the boundary markers as a performance measure. 
In the effector space, the different dependent variables were computed from half cycles of joint kinematics and EMG data. Movement time (MTE) was computed from the forearm pronation/supination angle using the same method as in task space. Angular velocity was used for the time to peak velocity (TPVE) computation.

For the dependent variables related to muscle activity, the root mean square (RMS) value of the normalised EMG signal was calculated in order to estimate the amount of muscular activation (Dorel, Couturier, and Hug 2008) for each half cycle and for each muscle (RMB for BR and RMP for PT). The RMS value was computed using Equation (10).

$$
\mathrm{RMS}=\sqrt{\frac{1}{n}\left(x_{1}^{2}+x_{2}^{2}+\cdots+x_{n}^{2}\right)}
$$

where $n$ is the number of normalised EMG values on the total duration of the half cycle and $x$ the corresponding EMG normalised data.

To estimate the level of muscular co-activation, we used the wasted contraction (Gribble et al. 2003) measure. For the agonist-antagonist muscle pair (BR and PT), we computed the wasted contraction at each sampling point and for the total duration of the half cycle as:

$$
\text { Wasted contraction }(t)=\frac{\text { Smaller trace }(t)}{\text { Maximum (Effective contraction) }},
$$

where

Smaller trace $(t)$ is the smaller normalised activity in each agonist-antagonist pair computed at the time $t$,

Effective contraction was computed as:

$$
\text { Effective contraction }(t)=\text { Larger trace }(t)-\text { Smaller trace }(t) \text {, }
$$

with

Larger trace $(t)$ is the larger normalised activity in each agonist-antagonist pair computed at the time $t$.

Maximum (Effective contraction) is the maximum value of Effective contraction signal over the entire half cycle.

Both the minimal (MIW) and maximal (MAW) levels of this wasted contraction were estimated for each ID.

\subsection{Statistical analysis}

Repeated measures analyses of variance (ANOVA) were used to test the effects of ID (i.e. ID $=2, \mathrm{ID}=2.5$, ID $=3$, $\mathrm{ID}=3.5$ and ID $=4$ ) and direction of movement (i.e. right-directed and left-directed) on each dependent variable. In addition, regression analyses were conducted on MTT and MTE to determine the degree of linearity as a function of ID. Significant effects were further characterised through a measure of effect intensity (EI) and Newman-Keuls post hoc tests were used when significance level $(p<0.05)$ was reached.

\section{Results}

In the present experiment, the forces of advancement were much larger than drag forces, whatever the experimental condition (Figure 3). Despite being representative of a realistic piloting situation, the control of the vehicle was consequently very close to a classical second-order control, consisting in the control of acceleration along each axis of displacement.

\subsection{Movement time}

\subsubsection{MTT and MTE regression analyses}

The regression analyses using MTT data indicated a linear relationship between MTT and ID $\left(R^{2}=0.97\right)$. The regression analysis for effector space data also indicated a linear relationship between MTE and ID $\left(R^{2}=0.97\right)$.

\subsubsection{Effector space}

The analysis indicated a main effect of ID on MTE $\left(F_{(4,36)}=24.01, p<0.05\right.$, EI $\left.=72.70 \%\right)$. Newman-Keuls test on ID indicated significantly greater MTE for ID $=3(1.62 \pm 0.17 \mathrm{~s})$ than MTE at IDs $2(1.53 \pm 0.15 \mathrm{~s})$ and $2.5(1.57 \pm 0.15 \mathrm{~s})$, while MTE was greater at ID $=4(1.75 \pm 0.16 \mathrm{~s})$ than at other IDs (Figure 4). No statistically significant effect of direction of movement on MTE was found. 


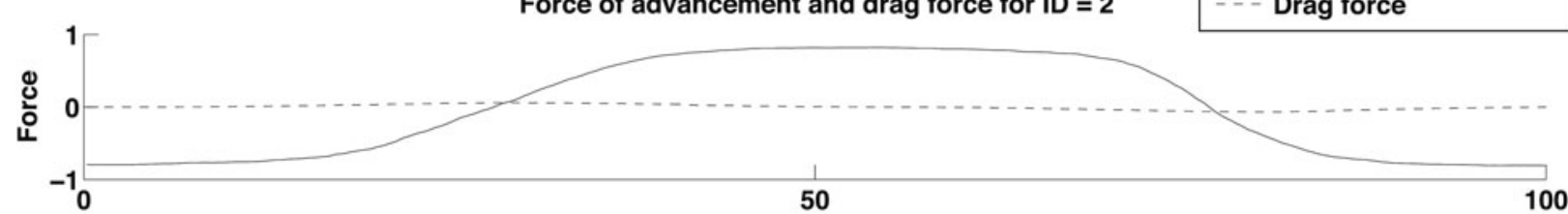

Force of advancement and drag force for ID $=2.5$
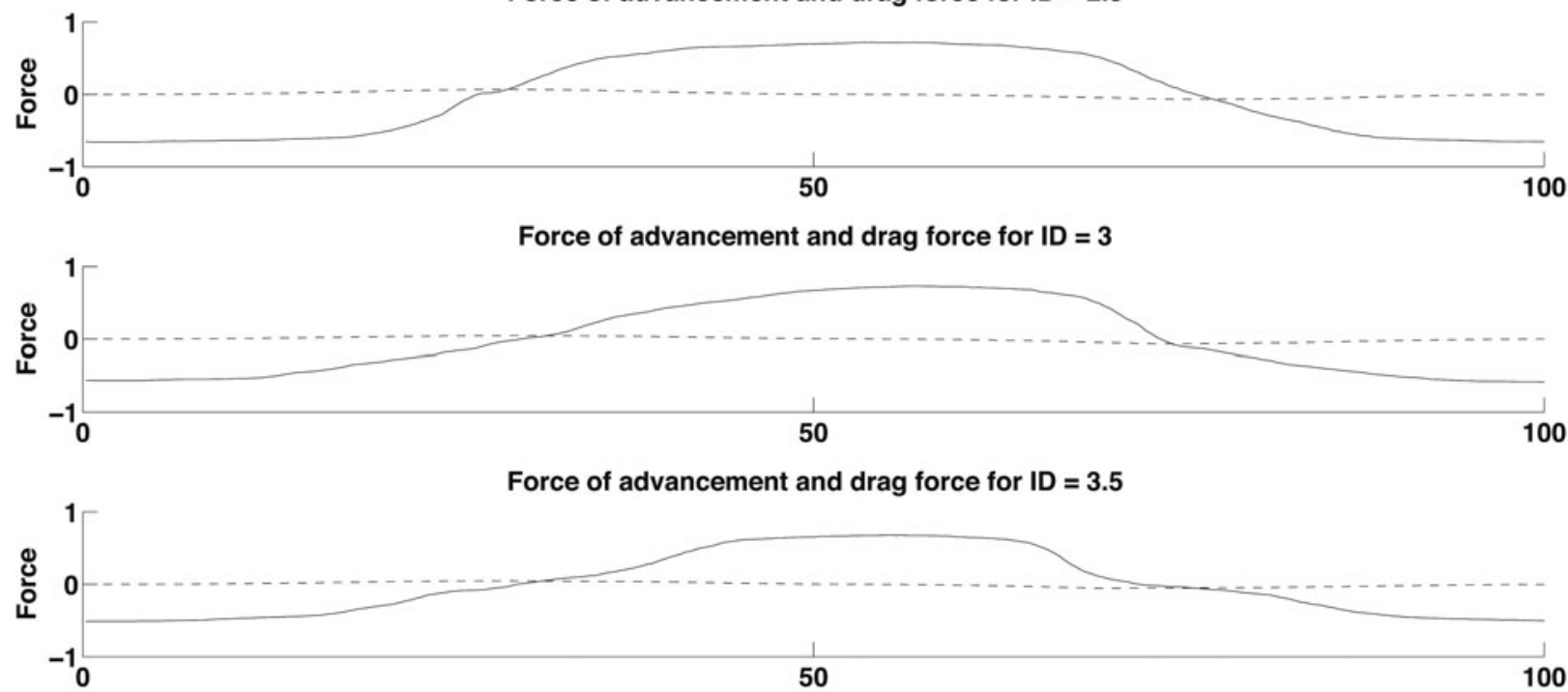

Force of advancement and drag force for ID $=4$

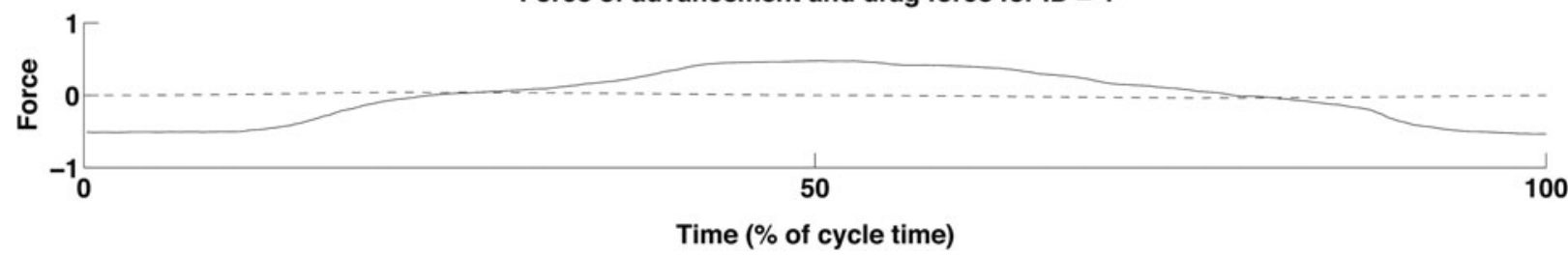

Figure 3. Force of advancement (solid line) and drag force (dotted line) along the lateral axis as a function of cycle time (\%) for each experimental condition (ID) and for one subject.

Note: Please note the low values of the drag force relative to the force of advancement.
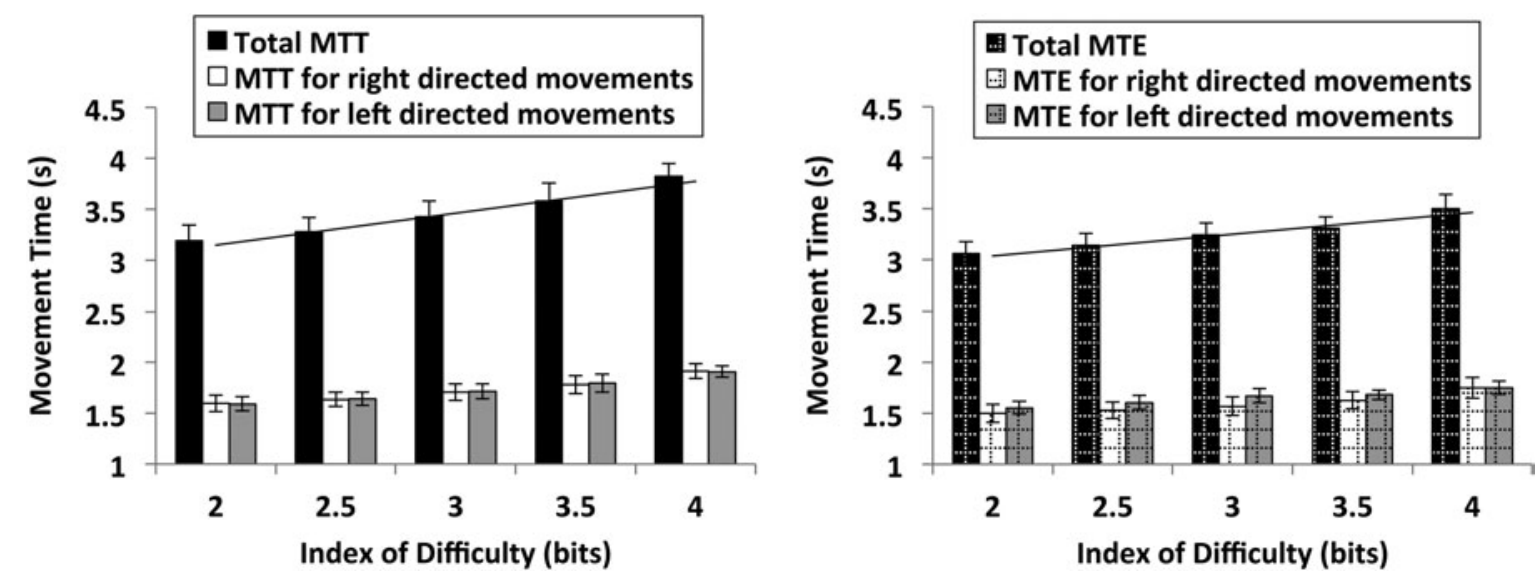

Figure 4. Average movement time in task space (MTT: left panel) and effector space (MTE: right panel) as a function of the index of difficulty for each direction of movement.

Note: The vertical bars indicate the standard deviation. 


\subsubsection{Task space}

The repeated measures ANOVA revealed significant main effect of ID $\left(F_{(4,36)}=96.10, p<0.05\right.$, EI $\left.=91.44 \%\right)$ on MTT. Newman-Keuls test on ID indicated that MTT for each ID was significantly different from all others (Figure 4). No statistically significant effect of direction of movement on MTT was found.

\subsection{Effective index of difficulty $\left(I D_{e}\right)$ in the task space}

The repeated measures ANOVA revealed significant main effects of ID $\left(F_{(4,36)}=57.60, p<0.05\right.$, EI $\left.=86.49 \%\right)$ on $\mathrm{ID}_{\mathrm{e}}$. Newman-Keuls tests on ID revealed that $\mathrm{ID}_{\mathrm{e}}$ significantly increased for each increase of ID except between ID $=3.5$ $(4.15 \pm 0.21$ bits $)$ and $\mathrm{ID}=4(4.30 \pm 0.34$ bits $)$ (Figure 5). No statistically significant effect of direction of movement on $\mathrm{ID}_{\mathrm{e}}$ was found.

\subsection{Index of performance (task space)}

The repeated measures ANOVA revealed significant main effect of ID $\left(F_{(4,36)}=7.40, p<0.05, \mathrm{EI}=45.12 \%\right)$ on IP. Newman-Keuls tests revealed higher IP at IDs $3\left(2.26 \pm 0.30\right.$ bits.s $\left.^{-1}\right), 3.5\left(2.36 \pm 0.30\right.$ bits.s $\left.^{-1}\right)$ and $4(2.28 \pm 0.31$ bits. $\left.\mathrm{s}^{-1}\right)$ than IP for ID $=2\left(2.10 \pm 0.41\right.$ bits.s $\left.^{-1}\right)$. In addition, IP was higher at ID $=3.5$ than at ID $=2.5(2.20 \pm 0.38$ bits. $\mathrm{s}^{-1}$ ) (Figure 6). No statistically significant effect of direction of movement on IP was found.

\subsection{Time to peak velocity}

\subsubsection{Effector space}

The analysis indicated a main effect of ID on TPVE $\left(F_{(4,36)}=3.76, p<0.05, \mathrm{EI}=29.50 \%\right)$. Newman-Keuls test on ID factor indicated similar TPVE for IDs 2 (50.17 $\pm 11.69 \%$ half-cycle time), 2.5 (48.11 $\pm 12.41 \%$ half-cycle time), 3 $(50.58 \pm 14.678 \%$ half-cycle time $)$ and 3.5 (43.58 $\pm 18.45 \%$ half-cycle time). The difference between TPVE was significant at ID $=4(37.35 \pm 21.08 \%$ half-cycle time $)$ with peak velocity appearing earlier in the cycle than at IDs $2,2.5$ and 3 (Figure 7). No statistically significant effect of direction of movement on TPVE was found.

\subsubsection{Task space}

The analysis indicated a main effect of ID on TPVT $\left(F_{(4,36)}=43.70, p<0.05\right.$, EI $\left.=82.90 \%\right)$. Newman-Keuls test indicated higher TPVT for IDs $2(51.78 \pm 1.12 \%$ half-cycle time $)$ and $2.5(50.80 \pm 1.48 \%$ half-cycle time) than for IDs 3

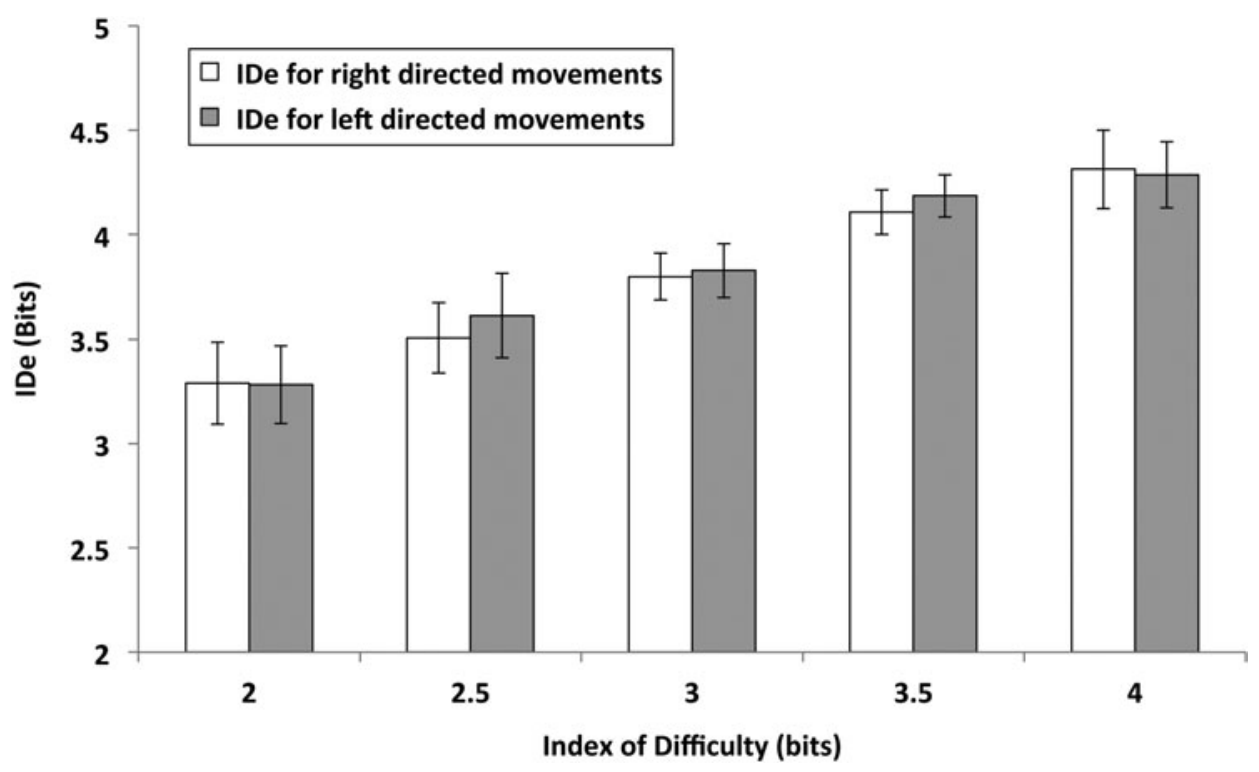

Figure 5. Effective index of difficulty in task space as a function of the index of difficulty for each direction of movement. Note: The vertical bars indicate the standard deviation. 


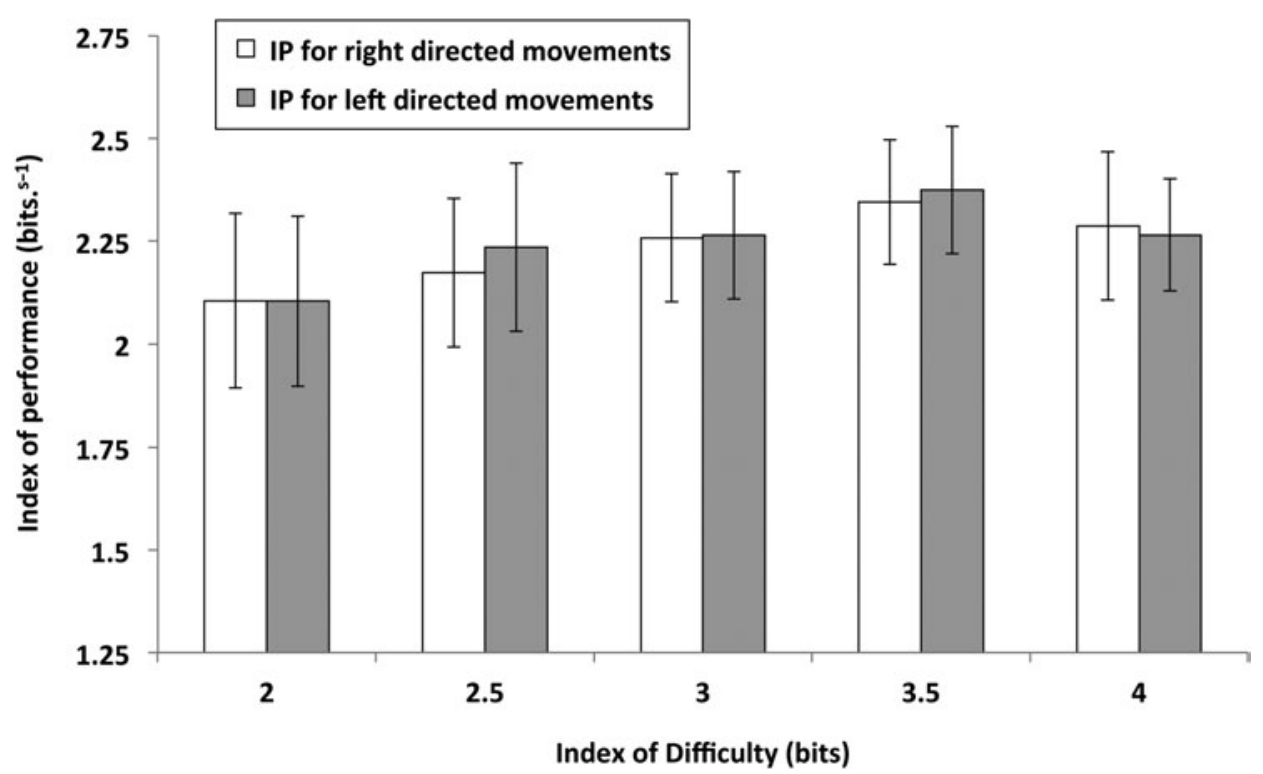

Figure 6. Index of performance in task space as a function of the index of difficulty for each direction of movement. Note: The vertical bars indicate the standard deviation.

$(49.50 \pm 2.21 \%$ half-cycle time), 3.5 (48.12 $\pm 2.7633 \%$ half-cycle time) and 4 (46.15 $\pm 3.38 \%$ half-cycle time). TPVT decreased for high task difficulty with TPVT for IDs 3, 3.5 and 4 significantly different from each other (Figure 7). No statistically significant effect of direction of movement on TPVT was found.

\subsection{RMS value of the EMG signal (effector space)}

ANOVAs revealed significant direction of movement $\times$ ID interaction for $\mathrm{RMB}\left(F_{(4,36)}=2.95, p<0.05, \mathrm{EI}=24.70 \%\right)$ and $\operatorname{RMP}\left(F_{(4,36)}=9.19, p<0.05, \mathrm{EI}=50.50 \%\right)$. The decomposition of the interaction into simple effects first showed main effect of ID on both RMB and RMP values. Post hoc analyses revealed that RMB of right- and left-directed movements significantly decreased for each increase of ID except between ID $=3.5$ and ID $=4$. Post hoc analyses also
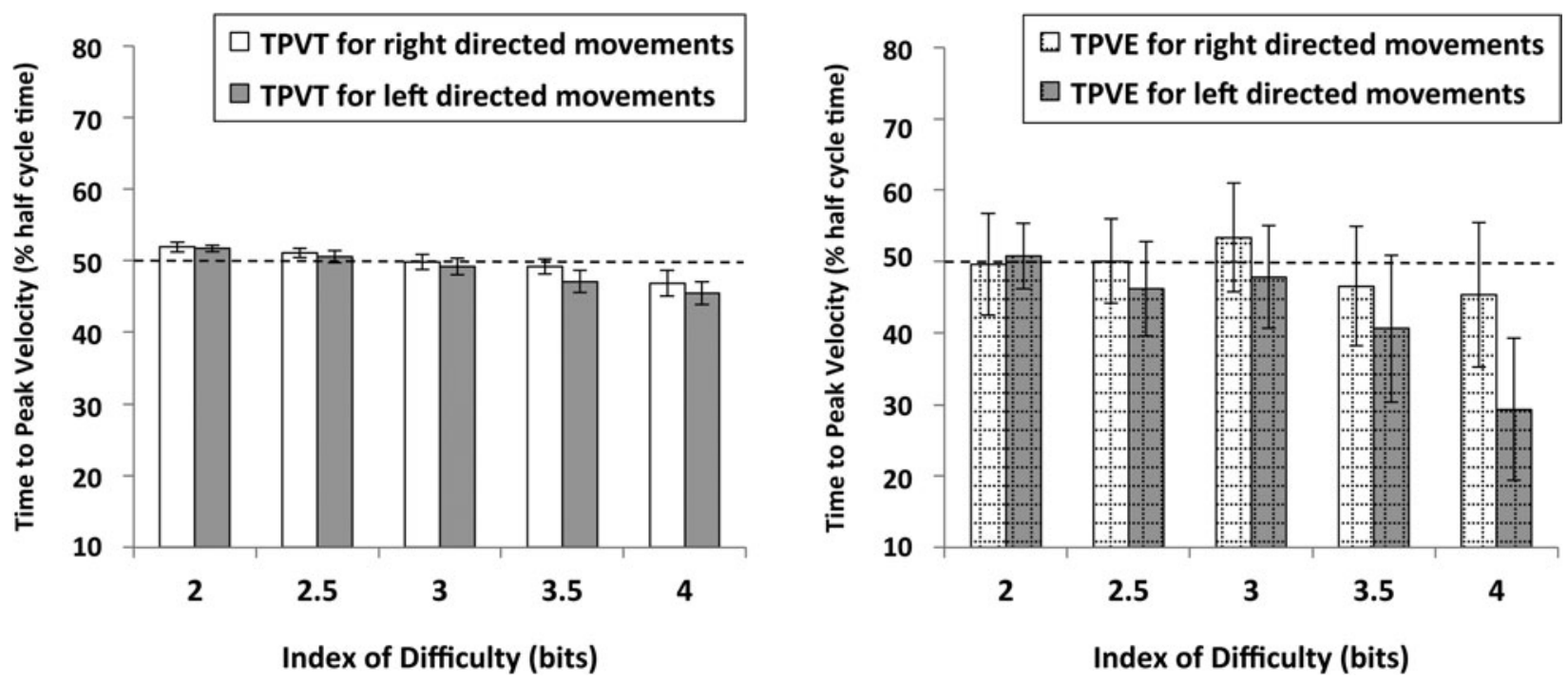

Figure 7. Time to peak velocity in task space (TPVT: left panel) and effector space (TPVE: right panel) as a function of the index of difficulty for each direction of movement.

Note: The vertical bars indicate the standard deviation. 

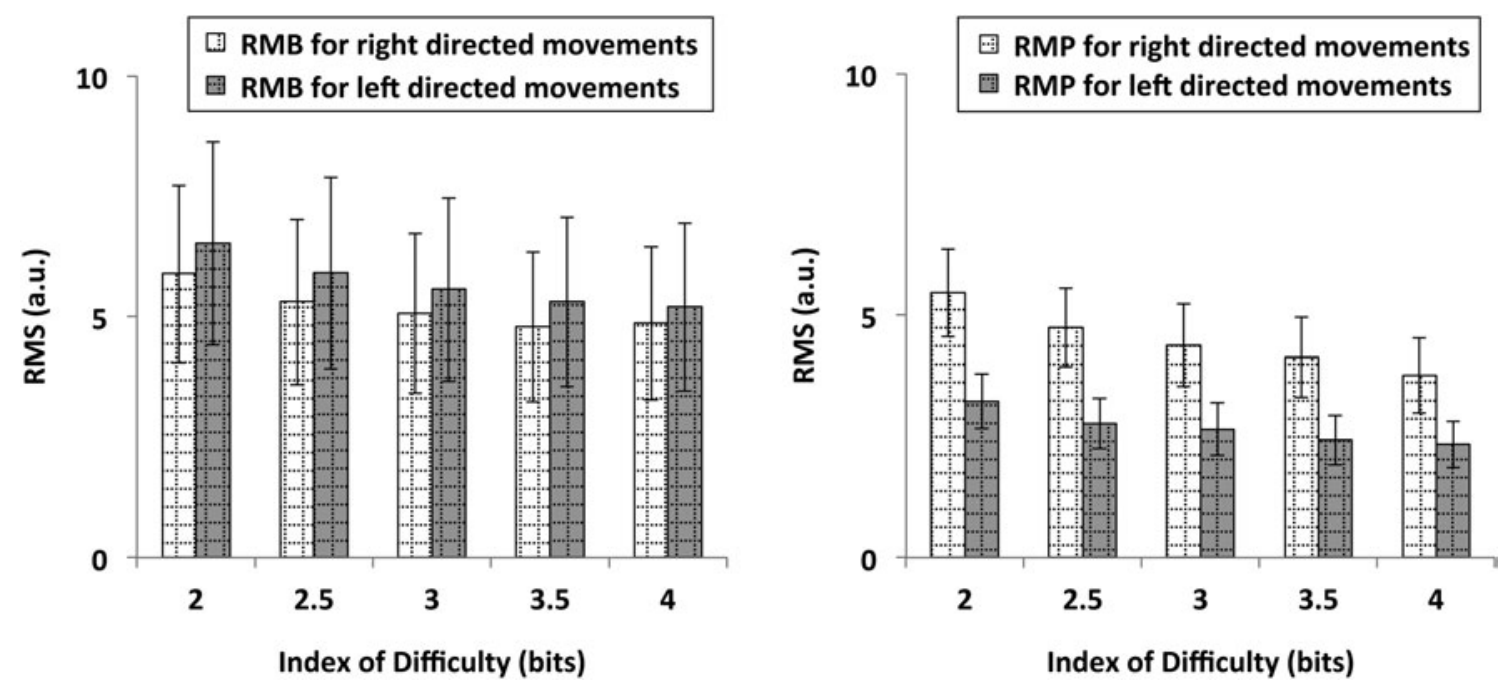

Figure 8. RMS value of the EMG signal in effector space for brachioradialis (RMB: left panel) and pronator teres (RMP: right panel) as a function of the index of difficulty for each direction of movement.

Note: The vertical bars indicate the standard deviation.

demonstrated that RMP values significantly decreased for each increase of ID when the movement was right-directed. When the movement was left-directed, RMP values significantly decreased when ID increased except between ID $=2.5$ and ID $=3$ and between ID $=3.5$ and ID $=4$. The decomposition of the interaction also showed main effect of direction of movement on both RMB and RMP with RMB higher for left-directed than for right-directed movements, while RMP was higher for right-directed than for left-directed movements (Figure 8). Finally, the analyses of the interaction for RMB and RMP demonstrated that the difference between right- and left-directed movements decreased as ID increased.

\subsection{Wasted contraction (effector space)}

\subsubsection{Minimal level}

The analysis detected a main effect of ID on MIW $(F(4,36)=6.30, p<0.05$, EI $=41.20 \%)$. Newman-Keuls test found higher MIW for ID $=4$ (34.69\% maximum effective contraction \pm 13.50$)$ than for all the other IDs $(28.64 \%$ maximum effective contraction \pm 12.94$)$. In addition, the main effect of direction of movement $(F(1,9)=6.73, p<0.05$, $\mathrm{EI}=42.80 \%$ ) was significant with MIW higher for right-directed than for left-directed wrist displacements (Figure 9).

\subsubsection{Maximal level}

No difference was seen for MAW, whatever the condition.

\section{Discussion}

The main objective of this study was to investigate the participants' performance during the use of an elastic pointing device under realistic inertial ISR. To achieve this goal, behavioural and biomechanical skills were analysed during an intensive aiming task in which different levels of accuracy constraints were manipulated. The task consisted in driving a vehicle-like virtual object along a slalom course as fast as possible under accuracy constraints. The participants controlled the displacements of the vehicle in the horizontal plane both along medio-lateral and longitudinal axes, while the displacement of the vehicle were related to the displacements of the device through a dynamical model.

We examined both the effect of accuracy constraints (task difficulty: ID) and of the direction of movement on MT, IP, the vehicle displacements (kinematic patterns), and on the electrical activity of the muscles and joint kinematics in the effector space.

Four key results were found: (1) MT as well as IP increased with accuracy constraints; (2) kinematic organisation changes with increasing accuracy constraints; (3) muscular activity decreased with task difficulty, whereas the level of minimal cocontraction increased with task difficulty; and (4) the muscular activity during the pointing task was affected by movement direction. 


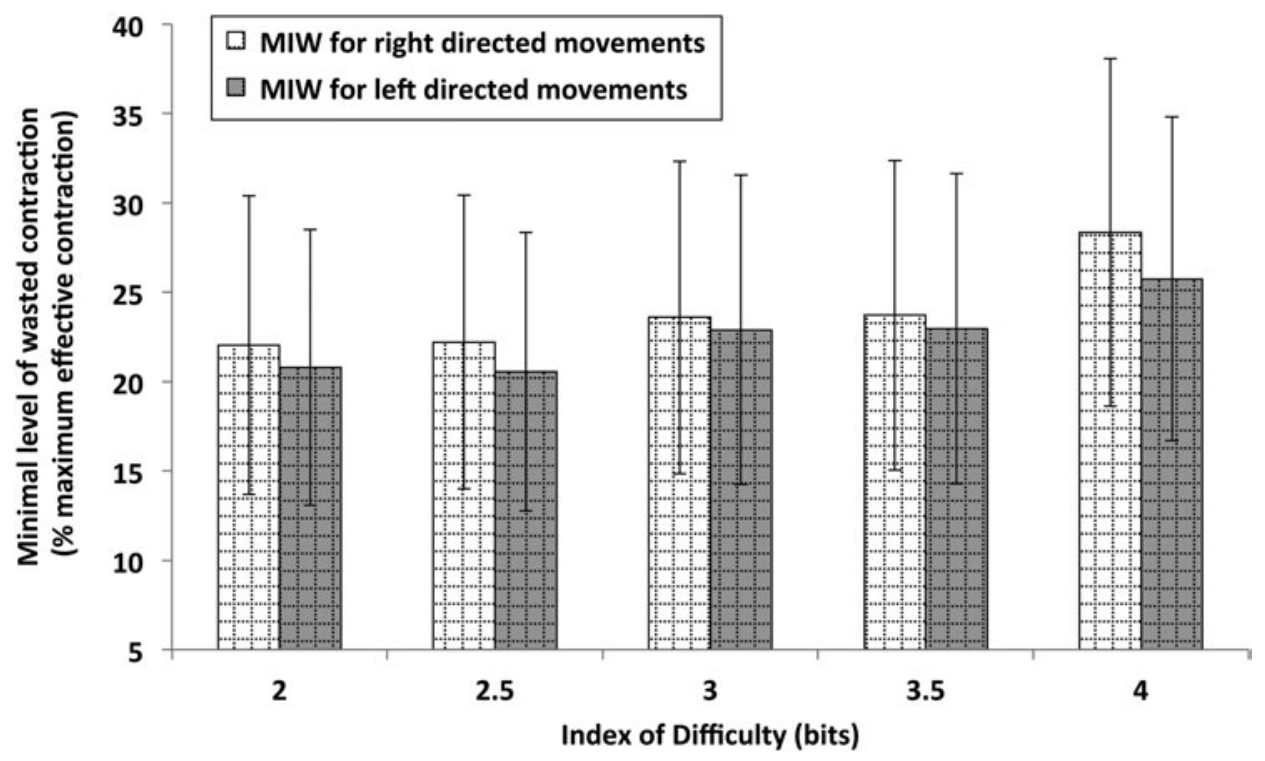

Figure 9. Minimal level of the wasted contraction of the agonist-antagonist BR-PT muscle pair in effector space (MIW) as a function of the index of difficulty for each direction of movement.

Note: The vertical bars indicate the standard deviation.

\subsection{Influence of ID and direction of movement on error rate, MT, kinematic organisation and IP}

Reciprocal aiming tasks often occur in intensive control situations such as aviation, industrial control, surgical robotics or video games. The vehicle of reference was an UAV. Considering the dynamics of the vehicle, the choice was made to use the same ISR for forward and lateral motions. This choice was supported by the inherent complexity of the task (2D control, non-linear ISR) and the desire to keep learning phases as short as possible.

Also, the Fitts experimental paradigm based on the Fitts' performance law is widely used by ergonomists as a standard for designing and evaluating input devices (ISO 2002). Contrary to previous linear ISR used for the evaluation of input devices (ISO 2002), the position of the virtual object obtained from the dynamical model in the present study was not linearly proportional to the joystick outputs, but resulted from a second-order control (i.e. force control).

In line with the initial work of Fitts (1954) and other previous studies (e.g. Bi, Li, and Zhai 2013; Mackenzie 1992b; Sasangohar, MacKenzie, and Scott 2009), the error rate was dependent on the ID (error rates ranged from 4.9\% at ID = 2 to $14.65 \%$ at $\mathrm{ID}=4$ ) (Figure 10). The average error rate of the trials among all the experimental conditions that were not rejected was equal to $10.27 \pm 5.26 \%$. Task instructions were, thus, well respected by the participants. Considering the

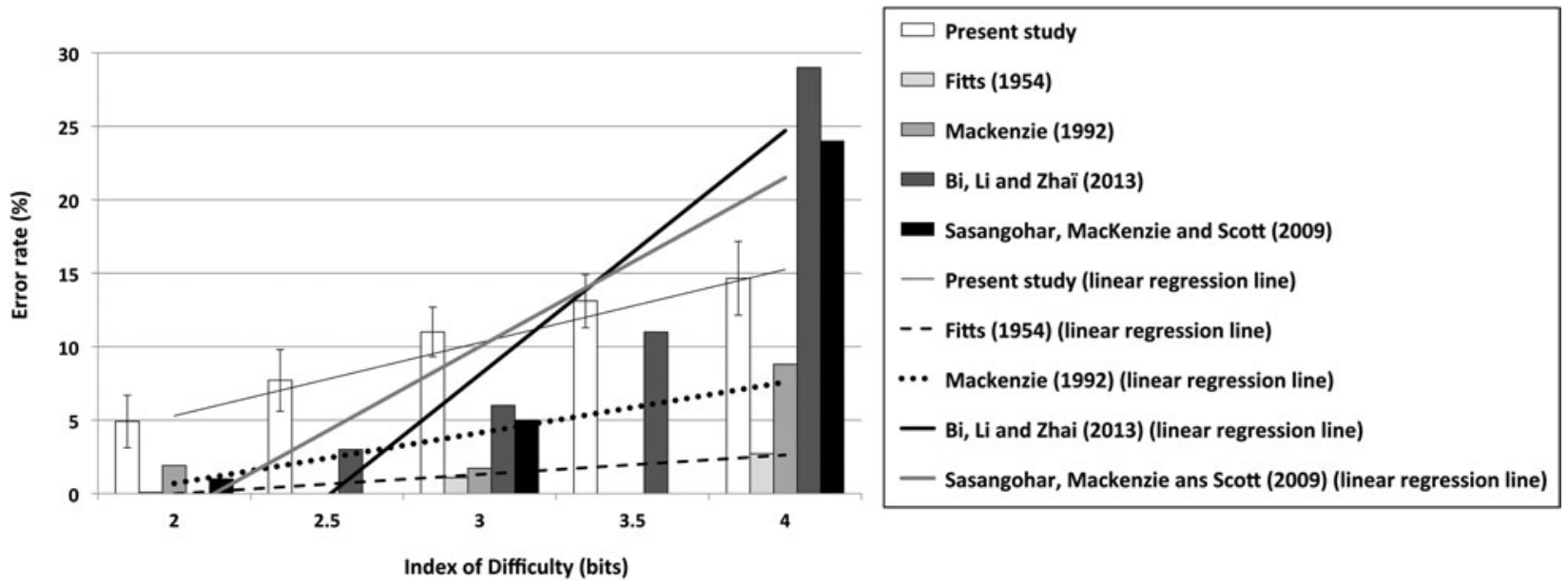

Figure 10. Error rates as a function of the index of difficulty for different Fitts' law studies.

Note: For each data set, the linear regression line is illustrated. 
average error rate and the influence of ID on the error rate, on MT (MTT and MTE), and on ID $\mathrm{e}_{\mathrm{e}}$ and IP, results support the idea that a valid speed-accuracy trade-off is at work in a realistic control situation. This main result is strengthened by the strong linear relationship between MT and ID for both MTE and MTT $\left(R^{2}=0.97\right)$.

The results of the present study confirmed that Fitts' law is quite universal and can be found even for very constraining tasks involving complex dynamics. The present experimental protocol combined a 2D slalom task with displacements being controlled along both axis and a non-linear realistic ISR. Indeed, an increase of ID, which was manipulated in the task space, induced an increase of MT both in the task and the effector spaces. Previous findings on 1D movements (Fernandez and Bootsma 2004) showed that Fitts' law can be retrieved in both spaces when the virtual object displacements (i.e. task space) are non-linearly coupled with the user's joint kinematics (i.e. effector space). The kinematic organisation of the pointing movement showed that an increase of ID also affected the kinematic patterns of the end effector. The analysis of the time to peak velocity revealed differences across different levels of difficulty. As ID increased, the peak of velocity occurred earlier with a deceleration phase longer than the acceleration phase (TPVE ranged from 50.17\% half-cycle time at ID2 to $37.35 \%$ half-cycle time at ID4 and TPVT ranged from 51.78\% half-cycle time at ID2 to $46.15 \%$ half-cycle time at ID4). These results are in line with the literature (Fernandez and Bootsma 2008; Mottet and Bootsma 1999) revealing that participants spent more time adjusting their movement based on sensory feedback when approaching the doors for high levels of difficulty (Fernandez and Bootsma 2004). In the reciprocal version of aiming task, as in the current study, functional description of this deceleration phase is more complex than in the discrete version of Fitts' task. Whereas in discrete aiming movements, the ballistic phase of movement is clearly distinguished from the visually controlled phase of movement while approaching the targets (Gan and Hoffmann 1988), this is not the case in the current investigated situation. During harmonic oscillations observed in reciprocal movements (Fernandez, Warren, and Bootsma 2006), participants have to simultaneously control the current deceleration when approaching a given target and prepare for the subsequent acceleration to move towards the next target. Regarding such differences, it has been proposed that successive reciprocal movements differ from the concatenation of discrete movements, thus making comparisons with discrete-based studies difficult (Guiard 1997; van Mourik and Beek 2004).

As expected, our results showed that the $\mathrm{ID}_{\mathrm{e}}$ increased with ID. Since $\mathrm{ID}_{\mathrm{e}}$ was computed from the effective width of the target (the movement actually done by the participants), one can consider that the lengthening of the deceleration phase described above assures a lower end-point variability to match with accuracy constraints. The IP, which is the combination of MTT and $\mathrm{ID}_{\mathrm{e}}$, presents the advantage of quantifying the performance in the control of a joystick and can thus be considered as a worthwhile dependent measure because it combines speed and accuracy in a single metric. Contrary to other methods of computation of the IP (based on the inverse of the slope of the linear regression line of the MT versus ID relationship), the method used in the present study where IP is computed for each $\mathrm{ID}_{\mathrm{e}}$ is not biased by the effect of the intercept of the MTT versus ID relationship (Soukoreff and MacKenzie 2004). Consequently, despite the fact that a positive intercept is graphically reported (Figure 4), IP - computed using the ISO standards - can be compared between experimental conditions and more generally between experimentations if the same ID range is used in the comparisons.

In line with previous studies (Douglas, Kirkpatrick, and MacKenzie 1999; Isokoski and Raisamo 2002; ; Poupyrev, Okabe, and Maruyama 2004; Silfverberg, MacKenzie, and Kauppinen 2001), IP was higher for higher ID with a 8.57\% increase in between ID2 and ID4 revealing a more efficient control with high accuracy conditions. However, the increase in IP is lower in the present study than in some other previous studies. For instance, Poupyrev, Okabe, and Maruyama (2004) reported a 23\% increase in IP between ID2.3 and ID6.3 and Silfverberg, MacKenzie, and Kauppinen (2001) reported a 59\% increase in IP between ID1.6 and ID5.1. Together with the limitations regarding IP computation, this last observation could also be attributed to differences between the 1D Fitts' paradigm used in the previously cited studies and the realistic adapted reciprocal aiming task proposed in the present work.

To summarise, the present study demonstrated that, in a 2D aiming situation with a non-linear ISR mimicking real inertial conditions, fast successive medio-lateral vehicle displacements presented similar kinematic characteristics to back and forth movements performed during classical 1D reciprocal aiming tasks. Participants adapted their movement to the accuracy constraints decreasing end-point variability and increasing both MT and error rate.

Pursuing the idea to propose conditions and/or protocol closer to ecological situation, we went further than the recommendations of the ISO standards by studying the effects of the direction of movement on reciprocal aiming performance. Previous results on discrete tasks showed no influence of direction (Oel, Schmidt, and Schmitt 2001; Whisenand and Emurian 1999). In the current study, we observed that in presence of a reciprocal representation of Fitts paradigm, MT and user's kinematic control of a joystick did not differ depending on the direction of movement regardless of the levels of ID and the space considered. Thus, IP was not affected by the direction of movement in agreement with HCI designer's predictions (ISO 2002).

The present study may help to evaluate the use of an input device in an intensive control situation, such as surgery or gaming by generalising Fitts' law applicability to more adapted and realistic conditions of use than previous ISO standards. 
Interestingly, and despite the control that has to be conducted along two directions, the Fitts' performance model was still confirmed when looking solely at the medio-lateral direction. Such a result corroborates previous findings from Hoffmann, Drury, and Romanowski (2011) who also highlighted a linear relationship between task difficulty (ID) and MT, whether the reciprocal aiming task was $1 \mathrm{D}$ or 2D. For the $1 \mathrm{D}$ reciprocal aiming task, MT was affected by accuracy constraints (target width or $W_{\mathrm{x}}$ ) on the axis of displacement along which successive opposite movements were required (axis defined as the direction of movement). In the 2D case, Hoffmann, Drury, and Romanowski (2011) showed that MT changes a little with the inclusion of further accuracy constraints along a second perpendicular axis (target height or $W_{\mathrm{y}}$ ) but was still strongly affected by $W_{\mathrm{x}}$. Consequently, the authors proposed that the most important task constraint is the required accuracy in the direction of movement (i.e. medio-lateral direction in the present study).

\subsection{Effect of ID and direction of movement on muscular activity}

Considering the muscle activity (EMG) of the user represents an additional way to characterise the performance in the use of a joystick. Our EMG-related results revealed a decrease in the amount of activation for BR and PT muscles (RMS) with the increase of ID (from 6.20 at ID2 to 5.03 at ID4 for BR and from 4.35 at ID2 to 3.05 at ID4 for PT). Since higher ID levels corresponded to a decrease in speed (increase of MT) for the user's forearm rotation and consequently for the medio-lateral vehicle displacements, the RMS was found to be directly related to the average speed in task and effector spaces.

These findings could be explained by the motor control mechanisms involved in a speed-accuracy trade-off task. The speed-accuracy trade-off is generally presented as the consequence of an optimal behaviour that guarantees the success of an aiming task in the presence of signal-dependent biological noise (i.e. noise whose variance increases with the size of the commands): faster movements require larger commands (muscle forces), and thus present more noise and variability (Auyang and Chang 2013; Guigon, Baraduc, and Desmurget 2008; Harris and Wolpert 1998; Meyer et al. 1988; Tanaka, Krakauer, and Qian 2006). Schmidt et al. (1979) were the first to highlight the role of biological noise in the control of movement accuracy showing that the end-point variability is inversely proportional to the movement speed. When the task tolerated a large variability of vehicle reversal position in task space (i.e. low ID), movement was produced with a high speed and large motor commands in effector space. For high levels of difficulty, movement speed and motor commands had to be reduced in effector space, so that variability of the vehicle position in task space remained within the door tolerance.

The way the participants modulated the co-activation of their upper limb agonist and antagonist muscles to control the displacements of the vehicle as a function of ID was also investigated. Measures of the minimal level of wasted contraction for the BR-PT muscle pair highlight the minimal joint stiffness required in the control of the upper limb stability to perform the slalom task. Increased muscular co-activation during movements not only induces more variable command signals as predicted from the presence of signal-dependent noise, but also improves limb stability and movement accuracy (Gribble et al. 2003; Huysmans et al. 2012; Laursen, Jensen, and Sjogaard 1998; Osu et al. 2004; Selen, Beek, and van Dieën 2006; Visser et al. 2004; Wong et al. 2009). Higher MIW was observed for movements that needed more accuracy at end-point location (higher ID). This confirms that the central nervous system would modulate co-activation for arm movements as a function of the accuracy constraints of the task (Gribble et al. 2003; Laursen, Jensen, and Sjogaard 1998; Osu et al. 2004; Visser et al. 2004). Of notable interest is that the control of the co-activation level seems independent of the activation level itself. Indeed, while the RMS decreased with ID, the co-activation increased. These results highlight the ability of the CNS to decouple both muscle force dedicated to set a joint in motion and muscle co-activation aimed at stabilising the joint.

Beyond these results, the distinction between right- and left-directed movements reveals some asymmetries at the level of muscular control. Interestingly and despite no difference due to direction of movement when investigating displacements in effector or task space, higher levels of BR-PT co-activation were obtained during right-directed wrist displacements (corresponding to forearm supination) although the joystick characteristics and the relationship between the angular position of the joystick and the vehicle displacements (i.e. dynamical model) were identical whatever the direction. Thereby, the way the participants modulated their muscle co-activation is different for supination and pronation motions. Similarly, we reported asymmetrical amounts of muscle activation with a significant effect of the direction of movement on RMS for the BR and the PT muscles. These last findings could be interpreted as the signature of asymmetrical co-activation strategies, consisting in specifically increasing the simultaneous activation of antagonist muscles according to the direction of movement. Neuromuscular responses are thus different between right- and left-directed movements during the use of a joystick. This asymmetry is caused neither by the ISR, nor by the mechanical characteristics of the joystick. At a biomechanical level, this asymmetry could rather be explained by forearm pronation movement that would be more closely related to the neuromuscular strategies used daily by the participant than by the forearm supination movement.

Thus, forearm supination would require more complex neuromotor adjustments from the sensory and motor systems (Borges et al. 2007). The results of the present work are in line with recent studies that suggested the influence of biomechanical constraints on muscle control of end-point position regarding the direction of movement (Hu, Murray, and 
Perreault 2012; Tee et al. 2010). These last authors used a musculoskeletal model of the human arm for investigating the control of end-point stiffness (i.e. relationship between perturbations applied at the hand and the forces generated in response) for different directions. End-point stiffness resisted perturbations in certain directions more strongly than in others because of specific neuromuscular responses.

It is interesting to note that the IP in the task space that was symmetrical do not inform us about the underlying asymmetrical motor performances of the user. Thanks to the current framework combining behavioural and biomechanical analysis, our results demonstrated that the consideration of the motor performance in the design of a joystick is relevant in order to make a better agreement between user's capabilities and their activities.

\section{Conclusion}

The current standard for evaluating computer input devices (ISO 2002) is limited to the analysis of the performance at a behavioural level, omitting kinematic organisation description as well as the motor activity of the users. Indeed, the ISO standard only proposes a questionnaire related to the comfort and effort levels to get information about the user's motor activity. The rationale of this study was to consider two different aspects of the user's performance (the behaviour in the task space and the motor activity in the effector space) in a realistic situation of speed-accuracy trade-off (adapted reciprocal aiming task, egocentric frame of reference, non-linear features of the ISR), providing complementary information for HCI designers about the effective use of an input device.

Our results concerning the motor performance made it clear that the neuromuscular responses were different depending on the action exerted on a joystick (i.e. the direction of movement), whereas the performance at a behavioural level was slightly affected. Since HCI designers only consider the performance at a behavioural level, the actual input devices present similar ISR regardless of the direction of the pointing movement. Consequently, the design of future ergonomics devices will have to meet the expectations of the neuromuscular system in order to facilitate their uses (i.e. reduction of the fatigue induced by the motor action, more intuitive use). In this perspective, future HCI designers and software/computer developers should develop devices that present asymmetrical ISRs and/or mechanical characteristics adapted to the neuromuscular requirements of the users.

\section{Note}

1. In classical Fitts' law studies, the error rate is generally kept under about 5\%. However, in the present study, participants performed a more complex task because (1) a non-linear ISR relating control input to device output was used (see experimental set-up) and (2) the nature of the aiming task involved control of displacements of the vehicle along two orthogonal axes simultaneously.

\section{References}

Auyang, A. G., and Y. H. Chang. 2013. "Effects of a Foot Placement Constraint on Use of Motor Equivalence during Human Hopping." PloS One 8 (7): e69429.

Bi, X., Y. Li, and S. Zhai. 2013. "FFitts Law: Modeling Finger Touch with Fitts' Law." In Proceedings of the 2013 ACM Annual Conference on Human Factors in Computing Systems, 1363-1372.

Borges, C. K., A. M. Rodrigues, J. F. Loss, R. D. S. Petersen, and M. A. Oliveira. 2007. "Effects of Elbow Joint Position on the Forearm Supination Torque Control in Young Adults." Brazilian Journal of Physical Therapy 11 (6): 487-493.

Boyle, J., S. Panzer, D. Wright, and C. H. Shea. 2012. "Extended Practice of Reciprocal Wrist and Arm Movements of Varying Difficulties." Acta Psychologica 140 (2): 142-153.

Card, S. K., W. K. English, and B. J. Burr. 1978. "Evaluation of Mouse, Rate-Controlled Isometric Joystick, Step Keys, and Text Keys for Text Selection on a CRT.” Ergonomics 21 (8): 601-613.

Casiez, G., D. Vogel, R. Balakrishnan, and A. Cockburn. 2008. "The Impact of Control-Display Gain on User Performance in Pointing Tasks." Human-Computer Interaction 23 (3): 215-250.

Dorel, S., A. Couturier, and F. Hug. 2008. "Intra-Session Repeatability of Lower Limb Muscles Activation Pattern during Pedaling." Journal of Electromyography and Kinesiology 18 (5): 857-865.

Douglas, S. A., A. E. Kirkpatrick, and I. S. MacKenzie. 1999. "Testing Pointing Device Performance and User Assessment with the ISO 9241, Part 9 Standard.", In Proceedings of the SIGCHI Conference on Human Factors in Computing Systems, $215-222$.

Elliott, D., S. Hansen, L. E. M. Grierson, J. Lyons, S. J. Bennett, and S. J. Hayes. 2010. "Goal-Directed Aiming: Two Components but Multiple Processes." Psychological Bulletin 136 (6): 1023-1044.

Fernandez, L., and R. J. Bootsma. 2004. "Effects of Biomechanical and Task Constraints on the Organization of Movement in Precision Aiming.” Experimental Brain Research 159 (4): 458-466.

Fernandez, L., and R. J. Bootsma. 2008. "Non-Linear Gaining in Precision Aiming: Making Fitts' Task a Bit Easier.” Acta Psychologica 129 (2): 217-227.

Fernandez, L., W. H. Warren, and R. J. Bootsma. 2006. "Kinematic Adaptation to Sudden Changes in Visual Task Constraints during Reciprocal Aiming." Human Movement Science 25 (6): 695-717.

Fitts, P. M. 1954. "The Information Capacity of the Human Motor System in Controlling the Amplitude of Movement." Journal of Experimental Psychology 47 (6): 381-391. 
Fitts, P. M., and J. R. Peterson. 1964. "Information Capacity of Discrete Motor Responses.” Journal of Experimental Psychology 67 (2): $103-112$.

Gan, K. C., and E. R. Hoffmann. 1988. "Geometrical Conditions for Ballistic and Visually Controlled Movements.” Ergonomics 31 (5): $829-839$.

Gribble, P. L., L. I. Mullin, N. Cothros, and A. Mattar. 2003. "Role of Cocontraction in Arm Movement Accuracy." Journal of Neurophysiology 89 (5): 2396-2405.

Guiard, Y. 1993. "On Fitts's and Hooke's Laws: Simple Harmonic Movement in Upper-Limb Cyclical Aiming." Acta Psychologica $82(1-3): 139-159$.

Guiard, Y. 1997. "Fitts' Law in the Discrete vs. Cyclical Paradigm.” Human Movement Science 16 (1): $97-131$.

Guigon, E., P. Baraduc, and M. Desmurget. 2008. "Computational Motor Control: Feedback and Accuracy." European Journal of Neuroscience 27 (4): 1003-1016.

Harris, C. M., and D. M. Wolpert. 1998. "Signal-Dependent Noise Determines Motor Planning." Nature 394 (6695): $780-784$.

Hermens, H. J., B. Freriks, C. Disselhorst-Klug, and G. Rau. 2000. "Development of Recommendations for SEMG Sensors and Sensor Placement Procedures." Journal of Electromyography and Kinesiology 10 (5): 361-374.

Hoffmann, E. R. 2012. "Contributions of Pre-Fitts Researchers to Goal-Directed Aiming Studies.” Journal of Motor Behavior 44 (1): $27-46$.

Hoffmann, E. R., and A. H. S. Chan. 2012. "Underwater Movement Times with Ongoing Visual Control." Ergonomics 55 (12): $1513-1523$.

Hoffmann, E. R., C. G. Drury, and C. J. Romanowski. 2011. "Performance in One-, Two- and Three-Dimensional Terminal Aiming Tasks." Ergonomics 54 (12): 1175-1185.

Hoffmann, E. R., and I. H. Sheikh. 2012. "Goal-Directed Aimed Movements with Path Obstructions." Ergonomics 55 (8): $946-962$.

Hu, X., W. M. Murray, and E. J. Perreault. 2012. "Biomechanical Constraints on the Feedforward Regulation of Endpoint Stiffness." Journal of Neurophysiology 108 (8): 2083-2091.

Huys, R., L. Fernandez, R. J. Bootsma, and V. K. Jirsa. 2010. "Fitts' Law is Not Continuous in Reciprocal Aiming." Proceedings of the Royal Society B: Biological Sciences 277 (1685): 1179-1184.

Huysmans, M. A., Marco J. M. Hoozemans, A. J. van der Beek, M. P. de Looze, and J. H. van Dieën. 2012. "Submovement Organization, Pen Pressure, and Muscle Activity are Modulated to Precision Demands in 2D Tracking." Journal of Motor Behavior 44 (5): 379-388.

ISO. February 15, 2002. "Reference Number: ISO 9241-9:2000(E). Ergonomic Requirements for Office Work with Visual Display Terminals (VDTs)-Part 9: Requirements for Non-keyboard Input Devices (ISO 9241-9)." International Organization for Standardization.

Isokoski, P., and R. Raisamo. 2002. "Speed-Accuracy Measures in a Population of Six Mice." In Proceedings of APCHI2002: 5th Asia Pacific Conference on Computer Human Interaction. Science Press, Beijing, China, 765-777.

Laursen, B., B. R. Jensen, and G. Sjogaard. 1998. "Effect of Speed and Precision Demands on Human Shoulder Muscle Electromyography during a Repetitive Task." European Journal of Applied Physiology and Occupational Physiology 78 (6): $544-548$.

Loeches De La Fuente, H., L. Fernandez, J. C. Sarrazin, E. Berton, and G. Rao. 2014. "Influence of Task Constraints and Device Properties on Motor Patterns in a Realistic Control Situation." Journal of Motor Behavior 46 (1): 1-15.

Mackenzie, I. S. 1992a. Fitts' Law as a Performance Model in Human-Computer Interaction. Toronto: University of Toronto.

MacKenzie, I. S. 1992b. "Fitts' Law as a Research and Design Tool in Human-Computer Interaction." Human-Computer Interaction 7 (1): $91-139$.

MacKenzie, I. S., and S. Jusoh. 2001. “An Evaluation of Two Input Devices for Remote Pointing.” Engineering for Human-Computer Interaction 2254: 235-250.

Meyer, D. E., R. A. Abrams, S. Kornblum, C. E. Wright, and J. E. Keith Smith. 1988. "Optimality in Human Motor Performance: Ideal Control of Rapid Aimed Movements." Psychological Review 95 (3): 340-370.

Mottet, D., and R. J. Bootsma. 1999. "The Dynamics of Goal-Directed Rhythmical Aiming." Biological Cybernetics 80 (4): $235-245$.

Oel, P., P. Schmidt, and A. Schmitt. 2001. "Time Prediction of Mouse-Based Cursor Movements." In Proceedings of Joint AFIHM-BCS Conference on Human-Computer Interaction IHM-HCI, 2, 37-40.

Osu, R., N. Kamimura, H. Iwasaki, E. Nakano, C. M. Harris, Y. Wada, and M. Kawato. 2004. "Optimal Impedance Control for Task Achievement in the Presence of Signal-Dependent Noise." Journal of Neurophysiology 92 (2): 1199-1215.

Poupyrev, I., M. Okabe, and S. Maruyama. 2004. "Haptic Feedback for Pen Computing: Directions and Strategies." In CHI' 44 Extended Abstracts on Human Factors in Computing Systems, 1309-1312.

Sasangohar, F., I. S. MacKenzie, and S. D. Scott. 2009. "Evaluation of Mouse and Touch Input for a Tabletop Display Using Fitts' Reciprocal Tapping Task." In Proceedings of the Human Factors and Ergonomics Society Annual Meeting, 53, 839-843.

Schmidt, R. A., H. Zelaznik, B. Hawkins, J. S. Frank, and J. T. Quinn Jr. 1979. "Motor-Output Variability: A Theory for the Accuracy of Rapid Motor Acts." Psychological Review 86 (5): 415-451.

Selen, L. P. J., P. J. Beek, and J. H. van Dieën. 2006. "Impedance is Modulated to Meet Accuracy Demands during Goal-Directed Arm Movements." Experimental Brain Research 172 (1): 129-138.

Silfverberg, M., I. S. MacKenzie, and T. Kauppinen. 2001. “An Isometric Joystick as a Pointing Device for Handheld Information Terminals.” In Proceedings of Graphics Interface 2001, 119-126.

Soukoreff, R. W., and I. S. MacKenzie. 2004. “Towards a Standard for Pointing Device Evaluation, Perspectives on 27 Years of Fitts' Law Research in HCI.” International Journal of Human-Computer Studies 61 (6): 751-789.

Tanaka, H., J. W. Krakauer, and N. Qian. 2006. “An Optimization Principle for Determining Movement Duration.” Journal of Neurophysiology 95 (6): 3875-3886. 
Tee, K. P., D. W. Franklin, M. Kawato, T. E. Milner, and E. Burdet. 2010. “Concurrent Adaptation of Force and Impedance in the Redundant Muscle System." Biological Cybernetics 102 (1): 31-44.

Van Galen, G. P., and W. P. de Jong. 1995. "Fitts' Law as the Outcome of a Dynamic Noise Filtering Model of Motor Control." Human Movement Science 14 (4-5): 539-571.

Van Mourik, A. M., and P. J. Beek. 2004. "Discrete and Cyclical Movements: Unified Dynamics or Separate Control?" Acta Psychologica 117 (2): 121-138.

Visser, B., M. P. De Looze, M. P. De Graaff, and J. H. Van Dieën. 2004. "Effects of Precision Demands and Mental Pressure on Muscle Activation and Hand Forces in Computer Mouse Tasks." Ergonomics 47 (2): 202-217.

Welford, A. T. 1968. Fundamentals of Skill. London: Methuen.

Whisenand, T. G., and H. H. Emurian. 1999. "Analysis of Cursor Movements with a Mouse." Computers in Human Behavior 15 (1): $85-103$

Wong, J., E. T. Wilson, N. Malfait, and P. L. Gribble. 2009. "Limb Stiffness is Modulated with Spatial Accuracy Requirements during Movement in the Absence of Destabilizing Forces." Journal of Neurophysiology 101 (3): 1542-1549.

Woodworth, R. S. 1899. “Accuracy of Voluntary Movement.” The Psychological Review: Monograph Supplements 3 (3): $1-106$.

Wright, C. E., and F. Lee. 2013. "Issues Related to HCI Application of Fitts's Law." Human-Computer Interaction 28 (6): $548-578$.

Wu, G., F. C. T. Van der Helm, H. E. J. Veeger, M. Makhsous, P. Van Roy, C. Anglin, J. Nagels, A. R. Karduna, and K. McQuade. 2005. "ISB Recommendation on Definitions of Joint Coordinate Systems of Various Joints for the Reporting of Human Joint Motion-Part II: Shoulder, Elbow, Wrist and Hand." Journal of Biomechanics 38 (5): 981-992.

Zhai, S. 1995. "Human Performance in Six Degree of Freedom Input Control." PhD thesis, University of Toronto.

Zhai, S. 2004. "Characterizing Computer Input with Fitts' Law Parameters - The Information and Non-Information Aspects of Pointing." International Journal of Human-Computer Studies 61 (6): 791-809. 